\title{
VELOCITY FLUCTUATIONS IN FORCED BURGERS TURBULENCE
}

\author{
Jean-Philippe Bouchaud ${ }^{1}$ and Marc Mézard ${ }^{2}$ \\ ${ }^{1}$ Service de Physique de l'État Condensé, Centre d'études de Saclay, \\ Orme des Merisiers, 91191 Gif-sur-Yvette Cedex, France \\ ${ }^{2}$ Laboratoire de Physique Théorique de l'Ecole Normale Supérieure*, \\ 24 rue Lhomond, 75231 Paris Cedex 05, France
}

\begin{abstract}
We propose a simple method to compute the velocity difference statistics in forced Burgers turbulence in any dimension. Within a reasonnable assumption concerning the nucleation and coalescence of shocks, we find in particular that the 'left' tail of the distribution decays as an inverse square power, which is compatible with numerical data. Our results are compared to those of various recent approaches: instantons, operator product expansion, replicas.
\end{abstract}

LPTENS preprint 96/37.

Electronic addresses : bouchaud@amoco.saclay.cea.fr, mezard@physique.ens.fr

${ }^{*}$ Unité propre du CNRS, associée à l'Ecole Normale Supérieure et à l'Université de Paris Sud 


\section{Introduction}

Burgers equation, which describes the potential flow of a fluid without pressure, provides a wonderful laboratory for testing new ideas and techniques in view of the study of fully developped turbulence in the Navier Stokes equation. These are two cases of non linear stochastic equations which share the same structure of the non linearity. The important difference comes from the nature of the large scale structures. In the case of Burgers equation these are shock waves and the corresponding physical picture of the flow is rather simple. This simplicity has already allowed for a very detailed study of the decaying turbulence [1, 2].

The forced case, in which the fluid is stirred randomly and steadily on large length scales, is more complicated. However it has been attacked recently by various methods like the operator product expansion [3], direct probabilistic methods [4, 5], instanton calculus[6] and replica method [7]. The latter method allowed to get a detailed solution in infinite dimension, and the finite dimensional solution seems to be within reach. This would be an important milestone for several reasons. It gives an example of a flow with strong intermittency, created by large scale structures. It provides a benchmark to test new -or older- ideas on fully developped turbulence. Furthermore this problem is also related to interesting problems in condensed matter physics, like the elastic lines in random media (e.g. vortices in superconductors), and growth problems [8]. In this respect it is interesting to notice that Burgers equation (with time playing the role of the running length scale) also appears naturally in the renormalization group study of manifolds in random media [9]. Therefore, the phenomenology of Burgers equation might be directly relevant to experimental studies of pinned Bloch walls [11] (or other elastic manifolds), besides its more direct potential applications to turbulence in one dimensional fluid flows [10] or to pattern formation in astrophysics [12].

In this paper we shall focus on a simple aspect of forced Burgers turbulence: the tails of the velocity gradient distribution in the regions where there are no shocks. We evaluate the 'right' tail through a rather simple computation, and compare it to the more sophisticated approaches developped recently [7, 3, 6]. We then give a conjecture on the 'left' tail which is based on a plausible argument, requiring the system to reach a stationnary state. We shall first discuss the one dimensional case, then turn to higher dimensions, and compare our results with the previously available ones.

\section{The slope dynamics in one dimension}

In one dimension we consider a velocity field $v(x, t)$ governed by Burgers equation:

$$
\frac{\partial v}{\partial t}+v \frac{\partial v}{\partial x}=\nu \frac{\partial^{2} v}{\partial x^{2}}+f(x, t)
$$

where $f(x, t)$ is a random force, which is supposed to have Gaussian distribution, with zero mean and a second moment:

$$
\left\langle f(x, t) f\left(x^{\prime}, t^{\prime}\right)\right\rangle=\epsilon \delta\left(t-t^{\prime}\right) R\left(\frac{\left(x-x^{\prime}\right)^{2}}{\Delta^{2}}\right)
$$

where $R$ is any smooth function decaying to zero fast enough at large arguments (e.g. an exponential as in [7]), $\Delta$ is the length scale of the stirring force and $\epsilon$ is the injected energy density. To keep consistency with the notations of our previous work [7], we fix the normalisations by requiring that at short distance $R(y)=1-\frac{3}{2} y+O\left(y^{2}\right)$.

Our approach is based on the following observation: even in the forced case, the flow is organised

in some smooth regions separated by shocks. Inside the smooth regions, the flow locally depends linearly on the position:

$$
v(x, t) \simeq \lambda(t)\left(x-x_{0}(t)\right)
$$


A linear expansion of the original equation allows to derive the evolution of $x_{0}(t)$ (which is irrelevant for our present discussion) and of the local slope $\lambda(t)$. This slope follows a Langevin equation:

$$
\frac{d \lambda}{d t}=-\lambda^{2}(t)+\eta(t)
$$

with a noise term $\eta$, due to the random stirring force, which has a Gaussian distribution, with:

$$
\left\langle\eta(t) \eta\left(t^{\prime}\right)\right\rangle=\frac{3 \epsilon}{\Delta^{2}} \delta\left(t-t^{\prime}\right) .
$$

This Langevin equation describes the relaxation of a particle at temperature $T=\frac{3 \epsilon}{2 \Delta^{2}}$ in a potential $V(\lambda)=\lambda^{3} / 3$. The zero temperature case corresponds to the decaying Burgers turbulence, where the slope decays in time as $\lambda(t)=\lambda_{0} /\left(1+\lambda_{0} t\right)$, leading to an asymptotic behaviour in which the slope is $1 / t$ independently of the initial conditions [1]. The forced case $T \neq 0$ leads to runaway solutions: starting from a positive slope $\lambda_{0}$, it will be eventually driven by the forcing to an infinitely negative slope, $\lambda \rightarrow-\infty$. This effect is nothing but the building up of a new shock, which tends to develop as soon as the fluctuations due to the forcing drive the system to a negative slope. However the tail of the slope distribution at positive $\lambda$ is unaffected by this effect (see below, Eq.(9)): it depends on rare noise configurations which, for a Gaussian forcing, lead to a Boltzmann form:

$$
P(\lambda) \sim C e^{-V(\lambda) / T}=C \exp \left(-\frac{2 \Delta^{2} \lambda^{3}}{9 \epsilon}\right), \quad(\lambda \rightarrow+\infty),
$$

where $C$ is a constant. A similar result was obtained in the context of pinned Charge Density Waves in [13].

Inside the linear regions, the velocity difference between two points at distance $r, u=v(x+r)-v(x)$, equals the slope $\lambda$ times the distance $r$, so that the slope fluctuations induce the following tail for the pdf of the velocity difference:

$$
P(u) \simeq \frac{C}{r} \exp \left(-\frac{2 u^{3} \Delta^{2}}{9 \epsilon r^{3}}\right), \quad(u \rightarrow+\infty)
$$

which is precisely the result derived in [3, 6]. We shall discuss the relationship of this elementary derivation with these other more elaborate ones later on.

From the evolution of the slope (舟) one can also discuss, in a more speculative way, the other tail of the pdf of the velocity differences (at large negative $u$ ). Since the regime we want to study is stationnary, the average number of shocks (or of linear regions) must be conserved. Hence, the slopes which 'disappear' at $\lambda=-\infty$ creating a new shock and thus two new slopes, must be compensated by the spontaneous disappearance of some other slopes through shock coalescence. In other words, for the number of 'cells' (locally linear regions) to be conserved, one must introduce a source term $J_{0} S(\lambda)$ in the corresponding Fokker-Planck equation, which describes these 'reinjection'/coalescence processes. These processes (and hence the correct form of $S(\lambda)$ ) are difficult to describe properly, but fortunately the asymptotic results are rather insensitive to the detailed shape of $S(\lambda)$. We normalize the integral over $\lambda$ of $S(\lambda)$ to 1, and write the Fokker Planck equation for the Langevin process (4) in the presence of the source term as:

$$
\frac{\partial P}{\partial t}=T \frac{\partial^{2} P}{\partial \lambda^{2}}+\frac{\partial}{\partial \lambda}\left(P \lambda^{2}\right)+J_{0} S(\lambda)
$$

This equation now possesses a stationnary solution with a non vanishing current $J_{0}$, which is nothing but the probability per unit time for a new shock to appear. This stationnary solution is given by:

$$
P(\lambda)=J_{0} \exp \left(-\frac{\lambda^{3}}{3 T}\right) \int_{-\infty}^{\lambda} d \lambda^{\prime} F\left(\lambda^{\prime}\right) \exp \left(+\frac{\lambda^{\prime 3}}{3 T}\right)
$$


where $F(\lambda)=\int_{\lambda}^{\infty} d \lambda^{\prime} S\left(\lambda^{\prime}\right)$ and $J_{0}$ is fixed by the normalisation of $P(\lambda)$. If we make the reasonable assumption that the reinjection term $S(\lambda)$ is a rapidly decaying function, the integral appearing in the above form (9) of the slope probability converges for $\lambda \rightarrow+\infty$, leading back the above result, Eq. (6). For $\lambda \rightarrow-\infty, F(\lambda) \rightarrow 1$, and the asymptotic form of $P(\lambda)$ is easily shown to be:

$$
P(\lambda) \sim \frac{J_{0}}{\lambda^{2}}, \quad(\lambda \rightarrow-\infty) .
$$

This in turn leads to a velocity difference distribution which decays as:

$$
P(u) \sim \frac{J_{0} r}{u^{2}}, \quad(u \rightarrow-\infty) .
$$

which happens to be precisely the form that Chekhlov and Yakhot have proposed using very different arguments, and which is compatible with numerical data [14].

Let us emphasize that the Fokker-Planck equation without the source term $S(\lambda)$ does not have a (normalizable) equilibrium state. This normalizable stationnary state can only appear in the presence of a non-zero current for large negative $\lambda$. The absence of an equilibrium state is also at the heart of the operator product expansion approach of Polyakov [3], on which we shall come back below.

\section{The slope dynamics in higher dimension}

The generalization of this approach to the $N$-dimensional Burgers equation is interesting. The velocity field verifies the equation:

$$
\frac{\partial \vec{v}}{\partial t}+(\vec{v} \cdot \vec{\nabla}) \vec{v}=\nu \nabla^{2} \vec{v}+\vec{f}(\vec{x}, t)
$$

where $\vec{v}$ is a gradient flow and the forcing term $\vec{f}$ is a random gradient field, with a Gaussian distribution of mean zero and second moment given by:

$$
\overline{f^{j}(\vec{x}, t) f^{k}\left(\vec{x}^{\prime}, t^{\prime}\right)}=\epsilon \delta\left(t-t^{\prime}\right)\left[\delta^{j k}-\frac{\left(\vec{x}-\vec{x}^{\prime}\right)^{j}\left(\vec{x}-\vec{x}^{\prime}\right)^{k}}{N \Delta^{2}}\right] G\left[\frac{\left(\vec{x}-\vec{x}^{\prime}\right)^{2}}{2 N \Delta^{2}}\right],
$$

where the $N$ dependance is chosen such as to insure the existence of a well defined large $N$ limit [7]. As before, the correlation $G$ decreases fast enough at large arguments and behaves as $G(y) \simeq 1-\frac{y}{2}+O\left(y^{2}\right)$ at small arguments.

Between the shocks (which now have a dimension $N-1$ ), the flow is locally radial, with a slope matrix $M$ :

$$
v_{j}(\vec{x}, t)=\sum_{k} M_{j k}(t)\left(x_{k}-x_{k}^{0}(t)\right)
$$

The slope matrix evolves in time as:

$$
\frac{d M_{j k}}{d t}=-\left(M^{2}\right)_{j k}(t)+y_{j k}(t)
$$

where $y_{j k}$ is a Gaussian random noise with a zero mean and a variance given by:

$$
\left\langle y_{i j}(t) y_{k l}\left(t^{\prime}\right)\right\rangle=\frac{\epsilon}{N \Delta^{2}}\left(\delta_{i j} \delta_{k l}+\delta_{i k} \delta_{j l}+\delta_{i l} \delta_{j k}\right) \delta\left(t-t^{\prime}\right) .
$$

We shall need to study the statistics of the eigenvalues $\lambda_{i}$ of the slope matrix $M$. From second order perturbation theory, one finds that these eigenvalues verify a Langevin type equation:

$$
\frac{\partial \lambda_{i}}{\partial t}=-\lambda_{i}^{2}+\frac{\epsilon}{N \Delta^{2}} \sum_{j(\neq i)} \frac{1}{\lambda_{i}-\lambda_{j}}+\eta_{i}
$$


where the level repulsion term has appeared [15], and the noise is Gaussian with a correlation:

$$
\left\langle\eta_{i}(t) \eta_{j}\left(t^{\prime}\right)\right\rangle=\frac{\epsilon}{N \Delta^{2}}\left(1+2 \delta_{i j}\right) \delta\left(t-t^{\prime}\right) .
$$

Compared to the one dimensional case, the situation is more complicated because of two effects: level repulsion on one hand, and correlation of the noises $\eta_{i}$ acting on each eigenvalue on the other hand. This noise correlation prevents the existence of an equilibrium (currentless) Boltzmann distribution, which one could use as above to obtain the right tail of the slope distribution. We shall instead use an instanton computation, which is well suited to deal with such correlations (see e.g. [16]).

We want to compute the pdf $P(u)$ of the longitudinal velocity difference,

$$
u=\sum_{i}\left(v_{i}(\vec{x}+\vec{r})-v_{i}(\vec{x})\right) \frac{r_{i}}{|\vec{r}|}
$$

which is given between the shocks by:

$$
u=r \sum_{i j} \hat{r}_{i} M_{i j} \hat{r}_{j}
$$

where $r=|\vec{r}|$ and $\hat{r}$ is a unit vector in the direction of $\vec{r}$. In terms of the eigenvalues of $M$, it takes the form:

$$
u=r \sum_{i=1}^{N} \lambda_{i} a_{i}^{2}
$$

where $\vec{a}$ is a random unit vector (obtained from $\hat{r}$ through a random rotation).

Let us introduce the Laplace transform of the pdf of the longitudinal velocity difference:

$$
G(\mu, r)=\int d u P(u) e^{\mu u}=\int_{-i \infty}^{i \infty} \frac{d z}{2 \pi i} \int d a_{1} \ldots d a_{N}\left\langle\exp \left(\mu r \sum_{j} a_{j}^{2} \lambda_{j}+z\left(\sum_{j} a_{j}^{2}-1\right)\right)\right\rangle
$$

In the spirit of recent works in turbulence [17, 18, 6] and in other fields [16, 19], we shall use a path integral representation for the probability distribution of the eigenvalues, and evaluate the large $\mu$ tail of $G(\mu, r)$ by finding the leading instanton trajectory. The path integral is written through standard manipulations [20]. The dynamics is started at a time $t_{0} \rightarrow-\infty$, and the eigenvalues are measured at $t=0$. We introduce conjugate fields $\hat{\lambda}_{i}(t)$ to implement the Langevin equation, and get after average over the thermal noise:

$$
G(\mu, r)=\int_{-i \infty}^{i \infty} \frac{d z}{2 \pi i} \int \prod_{j} d a_{j} \int \prod_{j} d\left[\lambda_{j}\right] d\left[\hat{\lambda}_{j}\right] \exp (-S)
$$

with an action:

$$
\begin{aligned}
-S & =\int_{-\infty}^{0} d t \sum_{j} \hat{\lambda}_{j}(t)\left(\frac{d \lambda_{j}}{d t}+\lambda_{j}^{2}(t)-\frac{\epsilon}{N \Delta^{2}} \sum_{k(\neq j)} \frac{1}{\lambda_{j}(t)-\lambda_{k}(t)}\right) \\
& +\int_{-\infty}^{0} d t \frac{\epsilon}{2 N \Delta^{2}}\left(2 \sum_{j} \hat{\lambda}_{j}(t)^{2}+\sum_{j, k} \hat{\lambda}_{j}(t) \hat{\lambda}_{k}(t)\right)+\mu r \sum_{j} a_{j}^{2} \lambda_{j}(t=0)+z\left(\sum_{j} a_{j}^{2}-1\right)
\end{aligned}
$$

The stationnarity conditions of this action are easily written. The effect of the parameter $\mu$ on the trajectories $\lambda_{j}(t), \hat{\lambda}_{j}(t)$ is just to impose the boundary condition $\hat{\lambda}_{j}\left(t=0^{-}\right)=-\mu r a_{j}^{2}$. The action $S$ has the following scaling property: under the change $\mu \rightarrow \gamma^{2} \mu, t \rightarrow \gamma t, \lambda_{i} \rightarrow \gamma \lambda_{i}, \hat{\lambda}_{i} \rightarrow \gamma^{2} \hat{\lambda}_{i}$ and 
$z \rightarrow \gamma^{3} z$, all the terms scale as $\gamma^{3}$, except the level repulsion term which is left invariant. Therefore, in the large $\mu$ limit, it is legitimate to neglect this level repulsion effect, and the following instanton solves these simplified equations:

$$
\begin{aligned}
a_{2} & =\ldots=a_{N}=\hat{\lambda}_{2}=\ldots=\hat{\lambda}_{N}=0 \\
\lambda_{1}(t) & =\frac{\phi}{1-\phi t} \quad \hat{\lambda}_{1}(t)=-\frac{\mu r}{(1-\phi t)^{2}}
\end{aligned}
$$

where the parameter $\phi$ is: $\phi=\sqrt{3 \epsilon \mu r /\left(2 N \Delta^{2}\right)}$. The action of this instanton is:

$$
-\ln (G(\mu, r)) \simeq S=-\sqrt{\frac{2 \epsilon}{3 N \Delta^{2}}}(\mu r)^{3 / 2}
$$

Assuming that this instanton gives the leading contribution to the Laplace transform $G$ at large $\mu$, one deduces from (26) the following tail of the pdf of velocity differences at large $u$ :

$$
P(u) \sim C \exp \left(-\frac{2 N \Delta^{2}}{9 \epsilon} \frac{u^{3}}{r^{3}}\right) \quad(u \rightarrow+\infty) .
$$

In order to check the dominance of the instanton solution (25), we have performed two checks: an evaluation of the contribution of other instantons, and a numerical simulation of the Langevin equation. Without level repulsion, other solutions can be found, with $(N-p) a_{j}$ and $\hat{\lambda}_{j}$ identically zero, and the

$p$ remaining $\lambda$ and $\hat{\lambda}$ all equal. The action of these instantons is a factor $f_{p}=\sqrt{\frac{p+2}{3 p}}$ smaller than (26). Since $f_{p}<1$ for all $p>1$, these other solutions can all be neglected. Taking into account the level repulsion can only increase further the action of these other solutions, so they should not contribute.

A direct numerical test of the result (27) is easily done. We have simulated the Langevin evolution equation for the eigenvalues (17) in the case $N=2$. We adopted a simple Ito discretization of time and computed the histogram of $u / r=\lambda_{1} \cos ^{2}(\psi)+\lambda_{2} \sin ^{2}(\psi)$ where $\psi$ is a random angle with uniform distribution in $[0,2 \pi]$. The time step has been kept adaptive in order to be able to use a small time step whenever the two eigenvalues are close to each other. Our simulation has been done at $\epsilon / \Delta^{2}=10 / 9$ and the statistics is over $10^{6}$ points. The result of the histogram is given in Fig.1, where the straight line gives the slope predicted from our instanton computation (27). The agreement confirms that the instanton (25) indeed gives the leading contribution. We have also simulated the Langevin process without the level repulsion term, and checked that we find the same tail for $\ln P(u)$. Let us just mention that the finite $u$ effects turn out to be larger in this latter case, and the analytic prediction for the tail is found only in the regime where $\ln P(u)<-10$. This finite $u$ corrections should also be important for the analysis of velocity difference tails in numerical simulations of Burgers equation.

Altogether, we are confident that the right tail of the velocity difference is given by (27) also in the multidimensional case. It is important to notice that this tail gets thinner and thinner as $N$ grows, and finally disappears in the limit $N \rightarrow \infty$, which was actually the situation that we considered in our previous paper []] using the replica method. Indeed, the calculation that we performed there shows that, for $N=\infty, P(u)$ is strictly zero when $u>u_{\Delta} \frac{r}{\Delta}$ (where $u_{\Delta}=(\epsilon \Delta)^{1 / 3}$ is the velocity at scale $\Delta$ ). This is compatible with Eq. (27) for $N=\infty$. It however clearly emphasizes the limitations of a large $N$ approach: although many features of the velocity field are correctly predicted, such a method is unable to predict the tails of the velocity distribution. Stated differently, the limits of large velocity difference and large dimension do not commute.

\section{Comparison with the direct instanton approach}

The previous results (6.27) on the tail of the longitudinal velocity difference at large positive $u$ are identical to those obtained in [3, 6] in one dimension and in [6] in dimensions $N>1$. The one 
dimensional result is thus confirmed by a very simple and direct derivation. In higher dimensions, we are unfortunately not able to solve fully for the probability distribution of the $N$ eigenvalues because of the noise correlations. We have rather found a simple instanton solution, which allows us to generalize the one dimensional result to arbitrary dimension. A naive generalisation of Eq. (8) to higher dimensions also suggest that the left tail of the distribution of velocity difference should decay as $u^{-2}$ in any finite dimension.

Although our instanton computation looks much easier than the one developped by Gurarie and Migdal [6] (we are dealing with the probability distribution of $N$ eigenvalues $\lambda_{i}$ instead of a $N$ component velocity field $\vec{v}_{i}(x)$ ), the two methods are actually very similar. In one dimension for instance, the instanton for the velocity field found in [6] corresponds precisely to a velocity field growing linearly with distance, with a slope called $\sigma$ in [6]. It is easy to deduce from the equations $(19,20)$ of [6] an evolution equation for $\sigma$ which is identical to the one which is derived from the stationnarity of (24). The same is true in larger dimensions.

To conclude our comparison with the work of [6], we have found that the positive $u$ tail of $P(u)$ derived from their nice instanton solution is certainly correct at least in one dimension. Our computation also indicates that this instanton cannot directly give the tail at negative $u$. In fact its structure, with a linear velocity field, shows that this instanton takes into account basically the linear regions, and not the shocks. As we argued before, these linear regions dominate the right tail of $P(u)$, but the left tail is given by a completely different process. In order to understand this left tail quantitatively, one needs to control both the formation of shocks, and the statistics of the sizes of the jumps in velocity at the shocks (or equivalently the statistics of the lengths of the linear regions). This has been achieved in the decaying problem [1], or in the forced case in large dimensions [7], and it is hard to think that this information is contained in a simple linear instanton configuration.

\section{Comparison with the operator product expansion}

Let us now discuss the operator product expansion (OPE) approach of Polyakov [3], which is supposed to give the full pdf $P(u)$ in the scaling region. Using some conjecture on the structure of the fusion rules in the OPE, one finds that, in one dimension, this $P(u)$ is exactly the totally assymmetric Lévy stable distribution of index $\frac{3}{2}$, given by the inverse Laplace transform of the function $G(\mu, r)$ found in (26). The behaviour of the right tail of $P(u)$ is again given by Eq. (6), and is thus compatible with the other approaches. However one should emphasize that this tail does not constitute a test for the fusion rules of the OPE. In the simple case of the 'two point' function $P(u)$, the OPE approach relies on the following steps. Restricting here to the case $N=1$, one first introduces a generating functional $Z\left(s_{1}, s_{2}, t\right) \equiv\left\langle\exp \left[s_{1} v\left(x_{1}, t\right)+s_{2} v\left(x_{2}, t\right)\right]\right\rangle$. In the stationnary regime, one should have $\frac{\partial Z}{\partial t}=0$, which, using the tricks of ref.[3], can also be written as:

$$
\frac{\partial^{2} Z}{\partial s_{1} \partial x_{1}}+\frac{\partial^{2} Z}{\partial s_{2} \partial s_{2}}-\frac{\partial Z}{s_{1} \partial x_{1}}-\frac{\partial Z}{s_{2} \partial x_{2}}=\left\langle\left[s_{1} f\left(x_{1}, t\right)+s_{2} f\left(x_{2}, t\right)\right] \exp \left[s_{1} v\left(x_{1}, t\right)+s_{2} v\left(x_{2}, t\right)\right]\right\rangle+\mathcal{A},
$$

where $\mathcal{A}$ is a term coming from the viscosity contribution, which is singular in the $\nu \rightarrow 0$ limit (the "anomaly"). Using the fact that the force $f$ is Gaussian, the first term in the right hand side can be reexpressed as:

$$
\frac{Z \epsilon}{2}\left[R(0)\left(s_{1}^{2}+s_{2}^{2}\right)+2 R\left(x_{1}-x_{2}\right) s_{1} s_{2}\right]
$$

Now, changing variables to:

$$
x_{1}=x+\frac{r}{2} \quad x_{2}=x-\frac{r}{2} \quad \mu=\frac{s_{1}-s_{2}}{2} \quad \mu^{\prime}=\frac{s_{1}+s_{2}}{2}
$$


and using translational invariance, one finds, for $\mu^{\prime}=0$, the following final equation for $G(\mu, r)=$ $\left\langle\exp \left[\mu\left(v\left(\frac{r}{2}\right)-v\left(-\frac{r}{2}\right)\right]\right\rangle:\right.$

$$
\left(\frac{\partial}{\partial \mu}-\frac{2}{\mu}\right) \frac{\partial}{\partial r} G=\frac{3 \mu^{2} \epsilon}{2 \Delta^{2}} r^{2} G+\mathcal{A}
$$

where $r<<\Delta$ is assumed. The simplest fusion rule leads to a contribution scaling as

$$
\mathcal{A} \simeq \alpha G+\frac{\beta}{\mu} \frac{\partial G}{\partial r}
$$

where $\alpha$ and $\beta$ are two constants to be determined. It turns out that the right hand tail of the solution $G(\mu, r)$ of (31) has a leading behaviour $\ln G \simeq \sqrt{2 \epsilon /\left(3 \Delta^{2}\right)}(\mu r)^{3 / 2}$ which is independent both of $\alpha$ and $\beta$ ! Therefore the $\exp \left(-c u^{3}\right)$ tail comes out exactly as what we derived in (7) (with precisely the same $c$ ), independently of the fusion rule. This is actually expected since we argued that this tail comes from the linear regions where one can completely neglect the anomaly. Without anomaly, the above equation has no solution which can be interpreted as the Laplace transform of a probability distribution (cf. the discussion after Eq. (11)). The fusion rules thus become crucial when one looks at the negative $u$ tail. If one insists that the solution $G(\mu, r)$ of (31) is the Laplace transform of a (normalizable) probability law, with an anomaly given by (32) with $\alpha=0$ for all $\mu$, one finds that $P$ is a Lévy distribution of index $\frac{3}{2}$, with a left tail decaying as $P(u) \sim|u|^{-5 / 2}$ [3]. This result disagrees with our above calculation, which suggested a $|u|^{-2}$ tail from the existence of a finite outgoing current at large (negative) slopes, related to the formation of new shocks. We do not have enough control on this 'reinjection process' of the slopes to make any strong statement. However, our argument is suggestive enough to try to look at other fusion rules which indeed lead to such a tail [21].

\section{Conclusion}

We have discussed the tails of the velocity difference distribution in Burgers' forced turbulence. We have shown how a direct method gives result which are in agreement with other methods in the right tail of the distribution. This method however suggests that the left tail is governed by the dynamics of shock formation, and requires some control over the shocks nucleation/coalescence processes. A simple hypothesis describing these processes in terms of an effective source term in the corresponding Fokker-Planck equation gives a $|u|^{-2}$ tail for the left side of the distribution, at variance with the simplest OPE conjecture, but apparently in agreement with numerical simulations.

It would be very interesting to extend these methods to describe more complicated quantities, such as the statistics of the velocity potential $h$ defined as $\vec{v}=-\vec{\nabla} h$. In particular, the statistics of the 'barrier heights' separating the different valleys of $h$ (corresponding to the cells of the Burgers flow) would be of direct interest to understand in detail the long time dynamics of randomly pinned objects!. In particular, it would be important to see to what extent the large $N$ result, which states that these barriers are exponentially distributed, has to be modified for finite $N$. Another interesting perspective is the generalization of these approaches to the case of a forcing correlated on long distances, where Kolmogorov's scaling is observed between the shocks [22, 23].

Acknowledgments. We want to thank A. Chekhlov, T. Dombre, V. Gurarie and V. Yakhot for interesting discussions.

\footnotetext{
${ }^{1}$ Conversely, as originally noticed by Feigel'man [13], the very large 'slope' tail $\lambda \rightarrow \infty$ (Eq. (6)) of the Burgers flow is related to the high frequency response of these pinned objects
} 


\section{References}

[1] J. M. Burgers, 'The Non-Linear Diffusion Equation', D. Reidel Pub. Co. (1974); S. Kida, J. Fluid. Mech. 93337 (1979); S. Gurbatov, A. Saichev, Sov. Phys. JETP, 53347 (1981).

[2] E. Aurell, U. Frisch, J. Lutsko, M. Vergassola, J. Fluid. Mech. 238467 (1992), S.E. Esipov, T.J. Newman, Phys. Rev. E 481046 (1993), S.E. Esipov, Phys. Rev. E 492070 (1994),

[3] A. Polyakov, Phys. Rev. E 526183 (1995).

[4] R. Kraichnan, in preparation.

[5] Y. Sinai, et al., Princeton University preprint (1996).

[6] V. Gurarie and A. Migdal, preprint hep-th 9512128, V. Gurarie, preprint (1996)

[7] J.P. Bouchaud, M. Mézard and G. Parisi, Phys. Rev. E 52 (1995) 3656.

[8] M. Kardar, G. Parisi, Y. Zhang, Phys. Rev. Lett. 56, 889 (1986); For a review, see: T. HalpinHealey, Y.C. Zhang, Phys. Rep. 254217 (1995) and references therein.

[9] L. Balents, J.P. Bouchaud, M. Mézard, 'The Large Scale Energy Landscape of Randomly Pinned Objects', to appear in J. Physique I (August 1996)

[10] H. Kellay and J. Rouch, preprint 1996.

[11] R. Vergne, J. C. Cotillard, J.L. Porteseil, Rev. Phys. Appl. 16449 (1981).

[12] Y. Zeldovitch, Astron. Astrophys. 884 (1972).

[13] M.V. Feigel'man, Sov. Phys. JETP 52555 (1980).

[14] A. Chekhlov and V. Yakhot, "Algebraic Tails of Probability Density Functions in the RandomForce-Driven Burgers Turbulence", in preparation.

[15] F. J. Dyson, J. Math. Phys. 13 (1972) 90, and refs. therein.

[16] A.J. Bray and A.J. McKane, Phys. Rev. Lett. 62493 (1989).

[17] M.J. Giles, Phys. Fluids, bf 72785 (1995).

[18] E. Balkovsky, G. Falkovitch, I. Kolokolov, V. Lebedev, preprint chao-dyn/9603015.

[19] For the use of this method in the context of fracture, see: M. Marder Phys. Rev. Lett.74 4547 (1995).

[20] C. de Dominicis, L. Peliti, Phys. Rev. B18 353 (1978).

[21] A. Polyakov, talk given at the IAS workshop on turbulence, May 1996.

[22] A. Chekhlov and V. Yakhot, Phys.Rev.E 51 (1995) R2739.

[23] F.Hayot and C. Jayaprakash, preprint (1996).

\section{Figure Captions}


Fig. 1 The logarithm of the probability distribution function of velocity differences, $\ln P(u)$, versus $(u / r)^{3}$, in dimension $N=2$, for $\epsilon / \Delta^{2}=10 / 9$. The curve is obtained from the simulation of the Langevin equation on the slope matrix eigenvalues, and the straight line indicates the prediction from the instanton computation (27). 


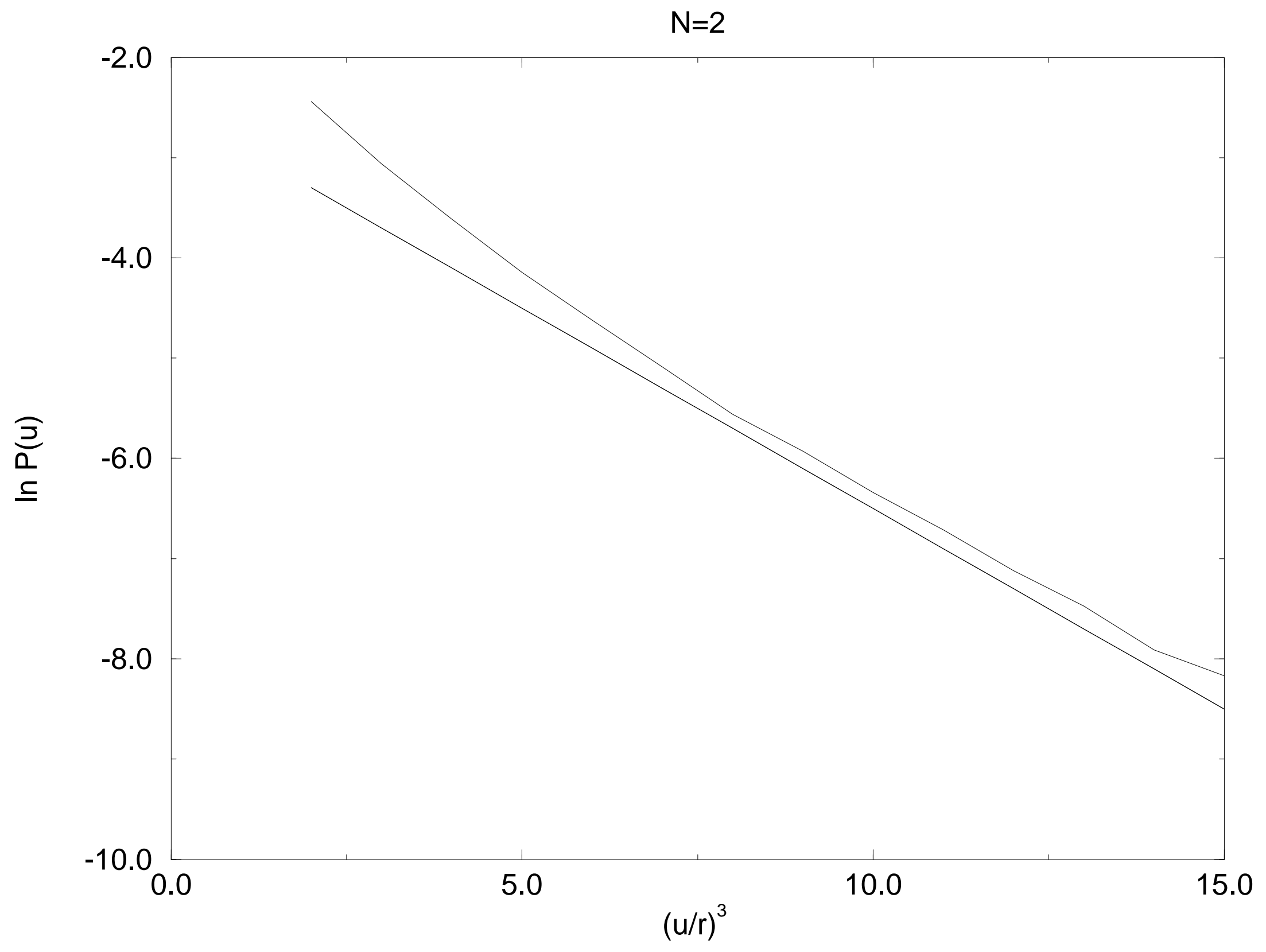

